\title{
Philosophiques
}

\section{Huet sceptique cartésien}

\section{José R. Maia Neto}

Volume 35, numéro 1, printemps 2008

Les valeurs de l'ironie (1) et le scepticisme à l'âge classique (2)

URI : https://id.erudit.org/iderudit/018247ar

DOI : https://doi.org/10.7202/018247ar

Aller au sommaire du numéro

\section{Éditeur(s)}

Société de philosophie du Québec

ISSN

0316-2923 (imprimé)

1492-1391 (numérique)

Découvrir la revue

\section{Citer cet article}

Maia Neto, J. R. (2008). Huet sceptique cartésien. Philosophiques, 35(1), 223-239. https://doi.org/10.7202/018247ar

\section{Résumé de l'article}

Pierre-Daniel Huet est un des sceptiques les plus importants de la fin du XVII siècle et du début du XVIII ${ }^{\mathrm{e}}$ siècle. Dans cet article, je cherche à montrer en six points que la principale source du scepticisme de Huet est paradoxalement Descartes, chaque point étant développé dans une section du texte : 1) Huet a découvert le doute cartésien avant de connaître le doute sceptique des anciens ; 2) le scepticisme du Traité Philosophique de la Faiblesse de l'Esprit Humain et l'anti-cartésianisme de la Censura Philosophiae Cartesianae faisaient originellement partie d'une même ouvrage ; 3 ) on trouve un Descartes sceptique dans la Censura ; 4) la biographie intellectuelle du Provençal dans le Traité Philosophique actualise et pyrrhonise la biographie intellectuelle du Descartes du Discours de la Méthode ; 5) quatre arguments sceptiques du Traité, dont le plus important de l'ouvrage, sont cartésiens ; 6) le scepticisme de Huet a été perçu par les premiers lecteurs du manuscrit du Traité comme partialement cartésien
Tous droits réservés @ Société de philosophie du Québec, 2008
Ce document est protégé par la loi sur le droit d'auteur. L’utilisation des services d’Érudit (y compris la reproduction) est assujettie à sa politique d'utilisation que vous pouvez consulter en ligne.

https://apropos.erudit.org/fr/usagers/politique-dutilisation/ 


\title{
Huet sceptique cartésien"
}

\author{
JOSÉ R. MAIA NETO \\ Universidade Federal de Minas Gerais \\ jrmaia@fafich.ufmg.br
}

\begin{abstract}
RÉSUMÉ. - Pierre-Daniel Huet est un des sceptiques les plus importants de la fin du XVII ${ }^{e}$ siècle et du début du XVIII ${ }^{e}$ siècle. Dans cet article, je cherche à montrer en six points que la principale source du scepticisme de Huet est paradoxalement Descartes, chaque point étant développé dans une section du texte : 1) Huet a découvert le doute cartésien avant de connaître le doute sceptique des anciens; 2) le scepticisme du Traité Philosophique de la Faiblesse de l'Esprit Humain et l'anti-cartésianisme de la Censura Philosophiae Cartesianae faisaient originellement partie d'une même ouvrage ; 3) on trouve un Descartes sceptique dans la Censura; 4) la biographie intellectuelle du Provençal dans le Traité Philosophique actualise et pyrrhonise la biographie intellectuelle du Descartes du Discours de la Méthode; 5) quatre arguments sceptiques du Traité, dont le plus important de l'ouvrage, sont cartésiens ; 6) le scepticisme de Huet a été perçu par les premiers lecteurs du manuscrit du Traité comme partialement cartésien.
\end{abstract}

\begin{abstract}
Pierre-Daniel Huet is one of the most important skeptics from the end of the 17th/begining of the 18th centuries. In this article, I show that Descartes is the main source of Huet's skepticism by means of six remarks, each developed in a section of the article. 1) Huet discovered Cartesian doubt before he discovered ancient skeptical doubt ; 2) the skepticism exhibited in the Traité Philosophique de la Faiblesse de l'Esprit Humain and the anti-cartesianism exhibited in the Censura Philosophiae Cartesianae were originally parts of the same work; 3) there is a skeptical Descartes in the Censura ; 4) the intellectual biography of the Provençal in the Traité Philosophique updates and pyrrhonizes Descartes's intellectual biography in the Discours de la Méthode; 5) four skeptical arguments in the Traité - including the most important one in the book — are Cartesian ; 6) Huet's skepticism was perceived as partially Cartesian by the first readers of the manuscript.
\end{abstract}

Bien que peu connu aujourd'hui, Pierre-Daniel Huet (1630-1721) occupait une place éminente dans la République des Lettres au XVII siècle. Expert dans les lettres classiques et sacrées (il a découvert à Stockholm un commentaire d'Origène sur l'Écriture, qu'il a ensuite traduit et édité), Huet a également été sous-précepteur du Dauphin (quand Bossuet en était le précepteur) qui, une

* Je remercie le $\mathrm{CNPq} /$ Brésil pour les moyens qu'il a mis à ma disposition pour cette recherche et Gianni Paganini, de l'Université du Piémont Oriental de Vercelli (Italie), pour la subvention qu'il m'a accordée pour pouvoir travailler sur Huet à Paris. Je remercie également le personnel de la Bibliothèque Nationale de France de m'avoir fourni les manuscrits et les livres ayant appartenu à Huet. Cet article est le fruit d'une série de conférences donnée en 2001 dans la section des sciences religieuses de l'École Pratique des Hautes Études. Je remercie le professeur JeanRobert Armogathe pour cette invitation, mais aussi pour les suggestions et remarques savantes qu'il m'a faites à cette occasion et qui ont nourri ma réflexion lors de la rédaction de cet article.

PHILOSOPHIQUES 35/1 — Printemps 2008, p. 223-239 
fois son éducation terminée, nommera son ancien maître évêque de Soissons, puis d'Avranches. Mais Huet a joué avant tout un rôle important dans les domaines philosophique et scientifique de son temps. Ainsi, il est à l'origine de l'établissement de la première Académie française des sciences soutenue par l'État en province, à Caen, au sein de laquelle il a fait plusieurs expériences scientifiques, et notamment des dissections anatomiques ${ }^{1}$. Dans le domaine de la philosophie, Huet est avant tout connu pour avoir été le principal critique de Descartes en France à la fin du XVII siècle. Son fameux livre Censura philosophiae cartesianae, publié en 1689, fut plusieurs fois réédité jusqu'au début du XVIII siècle et a suscité de nombreuses réactions importantes à l'époque, quelques-unes favorables, d'autres très critiques, telle la publication par Pierre-Sylvain Regis en 1691 d'une Réponse au livre intitulé Censura philosophiae cartesianae ${ }^{2}$. Le succès de cette critique anti-cartésienne proposée par Huet a très vite franchi les frontières de la France et on sait que l'ouvrage fit du bruit tant dans les universités allemandes et hollandaises, où le cartésianisme faisait débat, qu'italiennes ${ }^{3}$. Leibniz fut un des rares à avoir soutenu Huet à l'époque, comme le montre une lettre de Claude Nicaise à Huet, alors à Stockholm, datée du 10 juin 1691, dans laquelle Nicaise lui recopie une partie d'une missive qu'il a reçue de Leibniz le concernant:

J'honore infiniment Monseigneur l'évêque d'Avranches. Je suis vain d'apprendre qu'il se souvient de moi. Quelqu'un m'a dit que nous aurions bientôt de lui un ouvrage intitulé Concordia rationis et fidei. Tout ce qui vient de cette main est exquis et fera honneur à notre siècle auprès de la postérité. Un certain $\mathrm{M}$. Svelling de Brême ayant répondu à la censure de cet illustre évêque contre Descartes, on m'en demanda mon sentiment. Je repondis qu'à mon avis la meilleure façon de satisfaire à M. l'évêque d'Avranches serait de profiter des avis qu'il a donné aux cartésiens, de joindre les expériences de la physique, les recherches de la mathématique et la lecture des bons auteurs à celle de leur maître, et de se dépouiller d'une certaine vanité ridicule qui règne dans l'esprit de quelques-uns et qui les empêche d'avancer ${ }^{4}$.

La correspondance de Huet fait allusion à une possible collaboration entre les deux philosophes dans le débat anti-cartésien. Dans une lettre à Nicaise datée du $1^{\text {er }}$ avril 1697, Huet écrit en effet qu'il «attend avec impatience la promesse que [lui] fait M. Leibniz d'une liste de pilleries de M. Des Cartes ${ }^{5}$ ».

On voit bien comment Huet apparaissait à la fin du XVII ${ }^{e}$ siècle comme la référence européenne de l'anti-cartésianisme. Pour autant, les travaux philosophiques de Huet ne se sont pas limités à la polémique anti-cartésienne.

1. Voir Tolmer (1949) et Lux (1989).

2. Huet a laissé en manuscrit sa réponse à Régis: «La censure de la réponse faite par M. Regis au livre intitulé Censura philosophiae cartesianae ", manuscrit toujours inédit, que l'on peut consulter à la Bibliothèque Nationale de France.

3. Belgioioso (1999); Borghero (1998).

4. Pélissier (1889), p. 11.

5. Huet, Correspondance manuscrite de Nicaise (manuscrit). 
Dans le domaine de l'apologétique, où il était déjà connu par sa Demonstratio evangelica de 1679, il fait paraître en 1690 le livre mentionné plus haut par Leibniz sur le rapport entre foi et raison ${ }^{6}$. Mais l'important est ailleurs, dans l'élaboration d'un système philosophique, "son système " comme il l'appelle dans sa correspondance privée. Or, parce que ce système a une orientation sceptique, ce qui est peu recommandable pour un évêque censé défendre les dogmes chrétiens, Huet a préféré que le contenu en soit révélé après sa mort dans son Traité philosophique sur la faiblesse de l'entendement humain.

À partir d'une relecture de l'œuvre philosophique de Huet, j'aimerais soutenir ici une thèse apparemment paradoxale en montrant que, malgré son opposition farouche à Descartes sur laquelle les commentateurs s'entendent, tant l'origine que la nature du scepticisme huétien (du moins celui développé dans le Traité philosophique) ne sont pas classiques mais cartésiennes, ce qui est remarquable pour un érudit et un historien qui se dit très opposé au mépris de l'histoire et de l'érudition manifesté par Descartes et ses disciples, et notamment Malebranche ${ }^{7}$. On pourrait même dire que Huet développe un «cartésianisme sceptique » opposé au «cartésianisme dogmatique », position doctrinaire caractéristique de ce qu'on appelait à l'époque le cartésianisme. Si par le cartésianisme de l'époque, on entend non pas une adhésion aux thèses centrales de Descartes, mais le développement de philosophies dont la principale source est le travail de Descartes, on peut alors faire de Huet un cartésien au même titre que Malebranche, malgré l'opposition radicale de leurs philosophies. Dans cette perspective, Huet est cartésien au sens où les membres de la Nouvelle Académie (Arcésilas et Carnéade) étaient platoniciens, dans la mesure où ils se sont appropriés sceptiquement des positions qui, chez le philosophe qui en a été l'inspirateur (Platon pour Arcésilas, Descartes pour Huet), n'étaient pas sceptiques.

Afin de valider cette hypothèse, je propose six éléments qui lui confèrent une certaine plausibilité et laissent à penser que le scepticisme de Huet était bien cartésien. Ces éléments relèvent (1) de la biographie intellectuelle de Huet; (2) du projet philosophique dans lequel le Traité philosophique de la foiblesse de l'esprit humain a été conçu et écrit ; (3) d'une analyse interne de la Censura; (4) de la caractérisation par Huet du personnage qu'il a créé comme inventeur du système sceptique du Traité philosophique; (5) des arguments sceptiques du Traité; et (6) de la réaction des amis intimes anti-cartésiens de Huet à la lecture du manuscrit du Traité.

\section{Huet a connu le doute cartésien avant de connaître le doute sceptique}

Huet commence sa carrière d'érudit après sa sortie du collège jésuite de Caen, quand il s'associe avec Samuel Bochart, spécialiste reconnu de géographie sacrée. Pendant ses années de collège, Huet nous apprend dans ses mémoires

6. Huet (1690).

7. Lennon (2003). 
qu'il était très doué pour la géométrie, qui fut sa première passion intellectuelle ${ }^{8}$, avant qu'il ne découvre la philosophie moderne de Descartes et la philosophie des anciens. À cet égard, Huet rapporte dans ses mémoires la joie du jeune élève des jésuites occasionnée par la lecture des Principia Philosophiae qui venaient de paraître en 1644 : "Il me serait difficile de dire quel enthousiasme excitèrent en moi, jeune encore, et ne sachant rien des anciennes sectes philosophiques, la nouveauté de cette méthode et ces merveilles éblouissantes issues des principes les plus simples et les plus clairs ${ }^{9}$ ». Huet a donc été confronté en premier lieu au doute sceptique par la médiation des textes cartésiens. Il ne connaîtra le scepticisme de Sextus Empiricus qu'en 1661, dix-sept ans après avoir rencontré le doute cartésien ${ }^{10}$.

\section{Le scepticisme du Traité et l'anti-cartésianisme de la Censura comme parties originelles d'un même ouvrage}

La Censura fut publié en 1689, les Alnetanae quaestiones de concordia ratio et fidei en 1690, et le Traité en 1723, après la mort de Huet. Dans ses mémoires, Huet raconte que les deux premiers livres ont été écrits après la fin du souspréceptorat du Dauphin, quand il s'est installé dans l'abbaye d'Aulnay entre 1681 et 1684 :

Ces deux traités, et sur l'accord de la foi avec la raison, et contre la philosophie de Descartes, étaient des parties d'un plus grand ouvrage que j'avais le dessein d'écrire, et dont il n'est pas superflu de donner ici une esquisse. [...] Et comme l'esprit humain, renfermé dans des bornes étroites [...] et environné de ténèbres épaisses, essaie avec la raison de s'élancer vers la lumière et de toucher aux sommets ardus de la vérité, je résolus de chercher jusqu'à quelle hauteur il pourrait s'élever par ses propres forces et quel appui il devait emprunter à la foi. Cet exercice fut long et ne fut pas sans charmes, et mon ouvrage se développait, grossissait chaque jour davantage, lorsque je crus qu'il serait plus utile et que je m'accommoderais mieux à l'intelligence du commun des lecteurs, si je le divisais en parties et en chapitres. Telle est l'origine des Alnetanae quaestiones et de la Censura philosophiae cartesianae ${ }^{11}$.

8. Huet (1993), pp. 13-14: «[J]e passais les jours et les nuits à apprendre [la géométrie], et je sentais presque du dégoût pour les autres études qui m'avaient jusqu'alors si agréablement occupé. Je refusais même à la philosophie tous les soins que réclamaient la dignité et la grandeur de cette science. Tout enfin, à l'exception de la géométrie, me donnait des nausées ".

9. Ibid., p. 16.

10. Huet a connu le travail de Sextus Empiricus grâce à Louis de Cormis, politicien d'Aixen-Provence exilé à Caen; cf. Huet (1993), p. 90. Huet a pris connaissance de la Vie de Pyrrhon de Diogène Laërce et des Académiques de Cicéron avant de connaître Sextus. Il se dit en faveur des nouveaux Académiciens dans une lettre de 1660 (voir le « Recueil des lettres de Huet », manuscrit Bibliothèque Nationale, fonds latin 11432) et collabore à la traduction de Gilles Ménage des Vies des philosophes de Diogène Laërce en 1662 (voir le recueil des lettres de Huet, Bibliothèque Nationale, NAF 1341, fol. 106). On le voit, la lecture de Descartes a bien précédé celle des sources du scepticisme ancien.

11. Huet (1993), pp. 125-126. 
Nous avons découvert récemment à la Bibliothèque Nationale le plan de cet ouvrage original presque entièrement composé avant le découpage fait par Huet ${ }^{12}$. Ce plan, ainsi qu'un manuscrit annexe d'une première version d'un texte qui deviendra par la suite le Traité, confirme de manière définitive l'hypothèse avancée par certains chercheurs depuis le XIX ${ }^{e}$ siècle voulant que le texte du Traité date de cette même période ${ }^{13}$. Le titre de cet ouvrage est Quaestiones Alnetanea: non seulement il comprend les trois livres de l'ouvrage publié en 1690 sous ce titre, mais il en contient encore deux autres. Le titre du premier, «veritates ad humana mente per rationem certo cognosci non potest " n'est autre que le titre du premier livre du Traité: «La vérité ne peut être connue de l'entendement humain, par le secours de la raison, avec une parfaite et entière certitude ». Les livres II et III du Traité publiés en 1723 faisaient partie, avec le livre I, du premier livre de l'ouvrage indiqué dans le plan que nous avons découvert où ils figurent à titre de chapitres (et les chapitres du Traité apparaissent sous la forme de sections de chaque chapitre). Le deuxième livre du plan correspond exactement au contenu de la Censura publié en 1689, dont le titre dans l'ouvrage original était «mentis humana imbecillitas [titre de la version latine du Traité publiée en 1738] ad veritatem percipiendam comprobatur exemplo Cartesianae Philosophiae». Les livres III, IV et V correspondent aux trois livres publiés comme Quaestiones Alnetanea en 1690, à savoir: III) «de concordia rationis et fidei »; IV) «dogmatum christianorum et ethnicorum comparatio "; $\mathrm{V}$ ) " preceptorum christianorum et ethnicorum [...] comparatio ». On sait que Huet a montré le manuscrit de cet ouvrage original à des amis qui l'ont mis en garde en l'avertissant que la publication du premier livre sceptique (le Traité) lui causerait des ennuis et ruinerait sa réputation. Après avoir émis certaines résistances, Huet accepta à contrecœur de ne pas publier la première partie sceptique de son ouvrage ${ }^{14}$. Amputé de son premier livre, les quatre autres ne pouvaient plus paraître ensemble dans un même recueil et il fallait donc publier la critique du cartésianisme à part. En effet, la critique de Descartes ne pouvait plus être présentée comme une illustration de la philosophie sceptique du premier livre supprimé.

La Censura a initialement été écrite pour montrer que même la plus pertinente des philosophies, celle qui paraissait la plus acceptée à l'époque parce qu'elle représentait le plus grand effort de la raison pour «s'élancer vers la lumière et toucher aux sommets ardus de la vérité ", ne pouvait atteindre la certitude, et que seule la foi pouvait y conduire. Le schéma apologétique de Huet mis en jeu dans les livres originaux IV et V des Quaestiones Alnetanea (livres II et III des Quaestiones publiés en 1690) est une exégèse comparative

12. Bibliothèque Nationale, manuscrit fonds latin 11443.

13. La correspondance de Huet atteste également cette datation du Traité. Voir notamment les lettres publiées par Elena Rapetti (2003).

14. L'histoire dramatique des diverses versions et des tentatives frustrées de publication du Traité fera l'objet d'un autre travail. 
des religions ${ }^{15}$. Huet utilise sa vaste érudition pour essayer de montrer que toutes les religions et mythes païens sont des distorsions d'une seule religion originelle, le judaïsme. Cette stratégie peut être mise en parallèle avec le doute méthodique cartésien, comme l'a bien vu Richard Popkin. La vérité peut être retrouvée sous les croyances fausses des hommes, inscrite non pas dans la raison, comme le voulait Descartes, mais dans la Bible. Pour trouver la vérité, il faut par conséquent utiliser non pas la raison philosophique, mais l'érudition historique.

Traité, Censura, Concordia sont alors conçus par Huet comme les trois parties d'un même ouvrage. Le projet philosophique originel avorté de l'évêque d'Avranches peut alors être résumé de la façon suivante ${ }^{16}: 1$ ) sous la forme d'un règlement de compte avec le cartésianisme, d'abord dans le Traité, où est pensée la séparation entre la bonne partie du système (le doute méthodique), qui peut devenir utile à condition d'être sortie de son contexte philosophique cartésien, et la mauvaise partie (la métaphysique), qui fait obstacle au projet huétien et qui est attaquée dans la Censura; 2) sous la forme d'une conciliation sceptique (dans le sens de non philosophique) et érudite (historique) dans la Concordia d'une raison désormais privée de sa prétention à la vérité et remplacée par la foi chrétienne.

\section{Descartes sceptique dans la Censura}

La critique huétienne de Descartes est sceptique en deux sens. D'abord, dans le sens où Huet utilise les modes ou tropoi pyrrhoniens dans l'examen qu'il propose des doctrines cartésiennes, et notamment les cinq fameux tropoi du pyrrhonien Agrippa ${ }^{17}$. Ensuite, dans le fait que Huet valorise la partie «sceptique ", ou qui peut être ramenée vers le scepticisme, de la pensée cartésienne. La discussion portant sur le doute cartésien occupe presque un tiers de la Censura. La disposition de douter de tout propre à Descartes symbolise, pour Huet, la véritable disposition philosophique. Au début de sa démarche, Descartes adopterait donc la bonne méthode sceptique, mais il s'en écarterait malheureusement trop vite. À ce propos, dans l'article 14 du premier chapitre de la Censura, Huet fait allusion à la distinction que Descartes pose dans le Discours de la méthode entre son doute et le doute des sceptiques:

15. Voir Dupront (1930); Malbreil (1985) et Massimi (1985).

16. Nous sommes enclins à penser aujourd'hui que le cartésianisme a été une victime persécutée par les autorités religieuses, civiles et philosophiques de son temps, Huet apparaissant à cet égard comme une espèce de synthèse de tous ces types d'autorité. La correspondance de l'évêque d'Avranches montre plutôt que la situation était beaucoup plus complexe sur le terrain et que le cartésianisme avait quelques puissants soutiens, bien que discrets (on peut songer à Bossuet par exemple).

17. La relativité, la diaphonie (le conflit), l'hypothèse (affirmation sans justification), la régression à l'infini et le diallèle (circularité argumentative). À ce propos, voir Sextus Empiricus (1998), I, 164-177 et Diogène Laërce (1999), IX, 88-89. Huet déploie ces tropoi dans l'examen qu'il fait du cogito, de la règle générale (idées claires et distinctes) et des preuves de l'existence de Dieu. 
il a fixé et terminé tous les doutes à la connaissance certaine de ce principe 'je pense, donc je suis'. Quand il dit cela, il témoigne lui-même de son erreur puisqu'il ne commence proprement à errer qu'à partir du moment où il se sépare des sceptiques. Car, tout comme lui, ils ont bien vu qu'il fallait douter, mais avec cette différence que lui a cessé de douter lorsqu'il en avait le plus besoin, s'arrêtant à un principe qui n'est pas moins incertain que toutes les autres choses qui l'avaient engagé dans ses doutes, alors qu'eux continuent de douter parce qu'ils ne trouvent pas de principe solide sur lequel ils puissent s'appuyer avec une pleine certitude sans craindre d'être trompés ${ }^{18}$.

Descartes commence donc à philosopher en empruntant la méthode des sceptiques qui doutent de tout, mais il s'arrête en bon chemin là où précisément il fallait surtout douter, et ne poursuit pas par l'examen du cogito. Huet commence sa propre philosophie comme Descartes, en doutant de tout. Mais il s'écarte de Descartes au moment où Descartes s'écarte, lui, des sceptiques. La Censura essaie d'établir une hypothèse sceptique envisagée - pour être vite rejetée - par Descartes dans la fin de la Première Méditation et au début de la Seconde. Deux hypothèses se présentent après le doute hyperbolique: ou bien l'ego peut parvenir à quelque chose de ferme et solide (hypothèse établie par les Méditations), ou bien il doit établir avec certitude qu'il n'y a rien de certain. Cette deuxième hypothèse est la thèse centrale du traité philosophique: «la vérité ne peut être connue de l'entendement humain, par le secours de la raison, avec une parfaite et entière certitude ", titre du premier livre du Traité que " prouve » cette thèse à l'aide de treize arguments différents afin de montrer que la certitude parfaite n'est qu'une prérogative de la foi.

\section{La biographie intellectuelle du Provençal du Traité actualise et pyrrhonise la biographie intellectuelle du Descartes du Discours}

Contraint par des réactions très négatives à ne pas publier son système sceptique, Huet se décide à le traduire en français et élabore une fiction littéraire pour en cacher le véritable auteur. Le système devient alors celui d'un Provençal de Padoue qui présente oralement sa philosophie à un Théocrite de Pluvignac, Seigneur de la Roche, pseudo-auteur du Traité ${ }^{19}$. Parmi plusieurs modifications et additions au texte original de 1684-1685, Huet a ajouté une préface dans laquelle le Provençal raconte son parcours intellectuel qui l'a conduit au système qu'il va présenter au Seigneur de la Roche. Ce chemin sélectionne, intensifie et actualise quelques éléments "sceptiques" du discours autobiographique de Descartes dans le Discours.

Comme « la coutume de ce siècle » l'exige, le Provençal a «été élevé dans la Philosophie d'Aristote» et, tout comme Descartes, il s'est trouvé confronté

18. Huet (1689), I, 14, p. 42 (traduction nôtre).

19. Huet souhaitait que le livre soit publié sous ce pseudonyme seulement après sa mort. L'éditeur Du Sauzet publia le Traité deux ans après la mort de Huet mais sous le nom du vrai auteur, ce qui a fait scandale et entraîné une polémique sur l'authenticité de l'ouvrage. 
à « une $[\ldots]$ grande diversité d'opinions" qui le conduisent à être "fort choqué de ces disputes continuelles des Philosophes ${ }^{20}$ ». L'expérience sceptique de la diaphonie philosophique éprouvée par le Provençal coïncide avec celle vécue par Descartes à La Flèche: "Je ne dirai rien de la philosophie, sinon qu'il [...] ne s'y trouve encore aucune chose dont on ne dispute, et par conséquent qui ne soit douteuse ${ }^{21}{ }$. Le Provençal continue dans la même veine cartésienne en affirmant que la science pratiquée dans les écoles n'est "pas tant une recherche de la vérité, comme ils s'en vantaient, qu'une méthode de chicaner avec adresse, et de disputer subtilement ${ }^{22}{ }$.

Cette diaphonie philosophique pourrait être résolue, continue le Provençal, «si chacun [des philosophes], se dépouillant de ses préjugés, fût entré dans un nouveau et sérieux examen des dogmes, dont il parait si entêté [...] et qu'il eût appris une bonne fois à retenir sa créance, et à suspendre son jugement ${ }^{23}{ }^{\text {» }}$. Le discours du Provençal reprend à l'identique les termes du Discours: "pour toutes les opinions que j'avais reçues jusqu'alors en ma créance, je ne pouvais mieux faire que d'entreprendre, une bonne fois, de les en ôter ${ }^{24}$ ». La démarche du Provençal est bien celle du doute méthodique de Descartes, semblable à celle des sceptiques que Descartes a malheureusement, selon le Huet de la Censura, trop vite abandonnée en recourant à la certitude apportée par le cogito.

Et le Provençal ne cache pas l'influence de Descartes. D'emblée, il avoue qu'après avoir été déçu par la philosophie péripatéticienne, il a été attiré par le cartésianisme. Cette attirance n'a pas duré parce qu'il s'est vite aperçu "que les péripatéticiens se soutenaient encore; qu'il se formait de dangereuses factions contre Descartes; que Gassendi se faisait chef de parti ${ }^{25}$ ", et en a conclu que le cartésianisme ne faisait pas consensus. Puisque le cartésianisme n'a pas résolu le problème sceptique de la diaphonie, malgré l'intention affichée de Descartes en ce sens, le Provençal, qui, à ce moment précis, cherche encore, comme Descartes, "des fondements solides de la vérité ", se tourne alors vers le platonisme, philosophie où, une fois encore, il ne trouve « rien qui pût fixer [s]on esprit; nuls principes certains et déterminé ${ }^{26}{ }^{2}$. Lecture sceptique de Platon, que l'érudit Huet pouvait bien sûr puiser dans les Academica de Cicéron, mais dont la source la plus vraisemblable se trouve dans l'ouvrage qui a fasciné le jeune élève des jésuites. En effet, dans la lettre-préface à la traduction française

20. Huet (1974), pp. 3-4.

21. Descartes (1964), VI, p. 8.

22. Huet (1974), p. 4. Cf. Descartes (1964), VI, p. 69 : « je n'ai jamais remarqué [...] que, par le moyen des disputes qui se pratiquent dans les écoles, on ait découvert aucune vérité qu'on ignorât auparavant; car, pendant que chacun tâche de vaincre, on s'exerce bien plus à faire valoir la vraisemblance, qu'à peser les raisons de part et d'autre; et ceux qui ont été longtemps bons avocats, ne sont pas pour cela, par après, meilleurs juges ».

23. Huet (1974), p. 5.

24. Descartes (1964), VI, p. 13.

25. Huet (1974), p. 6.

26. Ibid., p. 7. 
des Principes, Descartes présentait déjà une interprétation sceptique de Platon en montrant que ce dernier, "suivant les traces de son maître Socrate, a ingénument confessé qu'il n'avait encore rien pu trouver de certain ${ }^{27}$ ».

Alors que Descartes dépasse à la fois le Platon sceptique et l'Aristote dogmatique (du moins si on en croit la préface des Principes) par ce qui, pour les sceptiques, n'est rien d'autre qu'un nouveau dogmatisme, le Provençal préfère en rester à la philosophie grecque et trouve enfin son bonheur dans la philosophie sceptique: «la doctrine d'Arcésilas, de Carnéade et de Pyrrhon me plût fort, et je jugeai qu'ils avaient mieux connu la nature de l'esprit humain que tous les autres philosophes ${ }^{28}$ ». La nature de l'esprit humain n'est pas de parvenir à la vérité du cogito, elle réside plutôt dans l'alternative envisagée par Descartes au début des Méditations où il évoque le cas où il ne trouverait rien de certain, envisageant la possibilité que l'esprit humain ne puisse connaître aucune vérité avec certitude, possibilité que Huet transforme en probabilité. Voilà la doctrine de base du système du Provençal qui finit son discours introductif en indiquant qu'il a découvert les fondements de son propre système, qui n'est pas cartésien, mais pas sceptique non plus puisqu'il ne suit pas l'enseignement de ces derniers «en plusieurs points ${ }^{29}$ ».

\section{Quatre arguments sceptiques du Traité, dont le plus important de tous ceux présentés, sont cartésiens}

Huet présente, dans le premier livre du Traité philosophique, treize "preuves» ou arguments visant à montrer que l'homme ne peut pas connaître la vérité avec certitude ${ }^{30}$. Les arguments mélangent des modes sceptiques anciens (ceux présentés par Sextus Empiricus) et modernes. Parmi les modernes, Descartes est la principale source sceptique de Huet. Des arguments tirés des textes cartésiens figurent dans quatre des treize preuves. Si on excepte deux preuves qui sont des arguments d'autorité (la première est tirée des auteurs sacrés, et l'autre, la treizième, évoque plusieurs éminents philosophes, grecs, juifs, arabes et orientaux qui ont adopté « la loi de douter»), la moitié ou presque des arguments proprement philosophiques de Huet sont d'origine cartésienne, y compris celui

27. Descartes (1964), IX, pp. 5-6.

28. Huet (1974), p. 8.

29. Ibid., p. 8. Deux de ces points sont l'abandon de la recherche de la vérité, puisqu'il sait que l'esprit humain ne peut pas la trouver, et l'abandon de la tranquillité comme fin de la philosophie. La finalité pyrrhonienne est substituée par deux autres fins: 1) éviter l'erreur et 2) "préparer l'esprit à recevoir la foi » (Ibid., p. 209).

30. Pour un examen de la composition du livre et une discussion de la spécificité du scepticisme qui y est présentée, voir Malbreil (1985). Sur le rôle du Traité dans la question du rapport entre raison et foi selon Huet, voir Quantin (1994). En ce qui concerne le rapport entre le scepticisme du Traité et le projet apologétique de Huet, voir Alberti (1978). Pour une conception du scepticisme du Traité comme réaction à Malebranche, voir Lennon (2005). 
que Huet indique comme étant le plus décisif ${ }^{31}$. On le voit, ou bien Huet interprète directement les positions cartésiennes en tirant des conséquences sceptiques de ces dernières, ou bien il s'approprie des lectures contemporaines sceptiques de Descartes, et notamment celle de Pascal ${ }^{32}$.

Les arguments d'origine cartésienne sont le second, le neuvième, le dixième et le onzième. Ce dernier est une utilisation sceptique de la doctrine cartésienne de la création des vérités éternelles, que Huet tire de la publication par Clerselier de la correspondance de Descartes ${ }^{33}$ :

Puis Descartes a cru et soutenu que Dieu peut changer l'essence des choses, et faire qu'elles ne soient pas ce qu'elles sont, en sorte que le nombre de vingt ne soit pas composé de deux dizaines, qu'un homme ne soit pas un animal raisonnable $[. .$.$] et partant, cette première proposition universelle, tout homme$ est un animal raisonnable, ne sera pas véritable ${ }^{34}$.

La doctrine de la création des vérités éternelles a captivé l'intérêt de Huet, qui a souligné dans son exemplaire des Lettres de Descartes les passages suivants de la page 110 du tome III :

Vous me demandez in quo genere causae Deus disposuit aeternas veritates. Je vous réponds que c'est in eodem genere causae, qu'il a creé toutes choeses, c'est-à-dire ut Efficiens \& Totalis Causa. Car il est certain qu'il est aussi bien Auteur de l'essence comme de l'existence des créatures. [...] Et je dis qu'il a été aussi libre de faire qu'il ne fut pas vrai que toutes les lignes tirées du centre à la circonférence fussent égales, comme de ne pas créer le monde : et il est certain que ces vérités ne sont pas plus nécessairement conjointes à son essence, que les autres créatures ${ }^{35}$.

31. Les arguments philosophiques non cartésiens sont les suivants: le troisième, qui utilise les cinq modes d'Agrippa dans la modalité aristotélicienne de la connaissance par genre et différence; le quatrième, qui évoque la doctrine du flux qu'on trouve dans le Théétète de Platon (cité par Huet) mais aussi dans l'Apologie de Raimond Sebond de Montaigne; le cinquième, qui se sert du deuxième trope d'Énésidème (Sextus Empiricus, 1998, I, 79-91) sur les différences entre les hommes, argument amplement utilisé par les sceptiques de la Renaissance et de la première moitié du XVII siècle; le sixième, qui évoque l'infinité des causes; le septième, qui pose le problème de la justification d'un critère de vérité (Ibid., II, 18-20); et le treizième, l'argument de la diaphonia philosophique, que Huet puise apparemment chez Sextus (puisqu'il cite la controverse des écoles médicales anciennes), mais qui est très répandu chez les sceptiques des $\mathrm{XVI}^{\mathrm{e}}$ et $\mathrm{XVII}^{\mathrm{e}}$ siècles, et qui se trouve aussi dans le Discours de Descartes, dans le passage qui inspire une strate de la biographie du Provençal.

32. On peut vérifier s'il s'agit d'une interprétation directe ou indirecte de Descartes en examinant la marginalia de Huet. Par exemple, les annotations insérées dans les Pensées montrent que Pascal a été décisif dans l'appropriation sceptique de l'argument cartésien du Dieu trompeur. Sur tout ceci, voir Maia Neto (2006).

33. Les lettres sur la création des vérités éternelles furent publiées dans le troisième volume des Lettres de Mr. Descartes. Huet indique la date de sa lecture du volume : 26 juin 1683, soit la période à laquelle il préparait ses Quaestiones Alnetanea originelles (manuscrit qui comprenait la Censura).

34. Huet (1974), p. 92.

35. La lettre est adressée à Mersenne et datée du 27 mai 1630; Descartes (1964), I, pp. 151152. 
Huet va proposer une utilisation sceptique et aussi dialectique de cette doctrine. Il est clair qu'il ne la présente ni comme vraie ni comme fausse. Un éventuel accord ne serait pas publiable par l'évêque. D'abord, parce qu'une telle concordance serait contradictoire avec le système sceptique mis en place dans le Traité. Ensuite, parce qu'il s'agit d'une doctrine amplement rejetée par les contemporains de Huet, par ses amis jésuites anti-cartésiens bien sûr (Louis le Valois), mais aussi par certains "cartésiens" (Malebranche et Leibniz).

Deux autres arguments cartésiens sont des scénarios sceptiques mis en place par la Première Méditation, à savoir celui du rêve (huitième preuve) et celui du Dieu trompeur (neuvième preuve). Ce dernier est médiatisé par la lecture de Pascal et l'argument de la "vraie religion ${ }^{36}$ ». L'argument du rêve n'est pas présenté comme cartésien et Huet ne mentionne pas le nom de Descartes dans ce chapitre, pas plus qu'il ne rappelle les sources sceptiques anciennes (les Académiques de Cicéron et les Hypotyposes de Sextus) ou modernes (l'Apologie de Montaigne) de l'argument. Par contre, Huet cite le Théétète de Platon, la plus ancienne source philosophique que nous ayons de l'argument, source directe des sceptiques anciens et de Montaigne. Le chapitre présente néanmoins des traces cartésiennes. D'abord, l'argument est employé pour mettre en doute l'existence d'un critérium indubitable permettant de discerner les perceptions que nous avons pendant la veille des perceptions que nous avons pendant le sommeil. Bien que ce problème soit mentionné dans le Théétète de Platon, il n'est pas question dans ce dialogue, comme cela le sera chez Descartes, de douter de la véracité de nos perceptions ${ }^{37}$. Deuxième trace cartésienne, l'argument est associé à une théorie de la perception sensible fortement inspirée des Passions de l'âme et du Traité de l'Homme de Descartes: "le cerveau peut être ébranlé, et [...] les esprits et les fibres peuvent être agités de la même sorte par des causes internes (le rêve), ou par des objets extérieurs ${ }^{38}$ ". La troisième trace cartésienne consiste en une allusion faite à la réfutation du scénario du rêve dans la Méditation VI. Descartes y allègue l'absence de continuité entre les expériences de rêve et de veille, argument contre lequel Huet évoque la possibilité d'une continuité fortuite, reprenant probablement la réponse apportée par Pascal au même argument de Descartes ${ }^{39}$.

Le quatrième argument sceptique huétien d'origine cartésienne est la seconde preuve, à savoir le fait que l'homme ne puisse "connaître avec une

36. Dans Maia Neto (2006), je montre également que Huet a souligné et annoté le texte de Pascal et repris son argument avec des mots quasi identiques dans le Traité.

37. Il serait trop long de montrer ce point ici. Il suffira simplement de remarquer que, dans le Théétète, l'argument présente la doctrine de l'homme mesure de Protagoras, construite par Platon, comme établissant la vérité de toutes les perceptions sensibles.

38. Huet (1974), p. 82. Je reviendrai sur cette théorie de la perception dans la considération du quatrième argument sceptique huétien inspiré de Descartes.

39. L'argument de Pascal se trouve dans la même section de l'édition de Port-Royal des Pensées : "Contrariétés étonnantes qui se trouvent dans la nature de l'homme à l'égard de la vérité » (Lafuma 131), reprise par Huet dans sa lecture sceptique de l'argument du Dieu trompeur. 
parfaite et entière certitude qu'un objet extérieur réponde exactement à l'idée qui en est empreinte en lui ${ }^{40}{ }^{\prime}$. L'argument est une application des dix modes pyrrhoniens à une théorie causale de la perception très marquée par le modèle mécaniste cartésien. Huet considère cet argument comme le plus décisif de son système, comme en témoigne le fait qu'il soit le seul retenu par le Provençal dans la synthèse du premier livre présenté au début du deuxième ${ }^{41}$.

Nous connaissons les choses par le biais des idées. Pour avoir connaissance d'une chose, il faut que l'idée dans l'âme soit semblable à son objet. Mais la théorie de la perception mise en place rend la correspondance entre l'idée et l'objet qui la cause très peu probable. Huet s'appuie sur la méthode sceptique des concessions successives au dogmatisme pour mettre en évidence la non-plausibilité de la correspondance entre idée et objet. Cette méthode est employée par Sextus Empiricus dans l'exposition des quatre premiers modes attribués à Énésidème, et reprise par Pierre Gassendi, qui emploie ces mêmes modes contre la théorie de la connaissance des aristotéliciens ${ }^{42}$.

Selon la théorie adoptée par Huet, la perception commence avec l'action de l'objet sur les sens qui reçoivent des traces de ce dernier. Ces traces sont ensuite mécaniquement transmises au cerveau par les esprits animaux à travers les nerfs. Les esprits animaux causent dans le cerveau une impression physique qui est enfin aperçue par l'âme unie au corps. On peut résumer cette théorie par le schéma suivant:

Objet $=>(1)$ milieu $=>(2)$ sens $=>(3)$ fibres du corps $=>(4)$ cerveau I (5) idée dans l'âme.

À chaque niveau de cet enchaînement, Huet indique, en s'inspirant des dix modes d'Énésidème, l'existence de variations physiques qui rendent plausible une très grande variation perceptive. La conséquence en est qu'on ne peut discerner une perception vraie de perceptions fausses, puisque toutes résultent du même processus.

(1) Le premier niveau est douteux à cause de la diversité des milieux. Huet applique ici le cinquième mode: le même objet apparaît différemment selon les différentes positions, distances et lieux où il est aperçu ${ }^{43}$. Par exemple, « [1] a couleur que l'on voit au soir dans les objets est différente de celle qu'on y voit au midi »; "le bleu de la mer change selon la diversité du vent, et devient quelquefois de couleur de pourpre et quelquefois jaune ${ }^{44}$ ».

(2) En supposant que les variations du milieu n'interviennent pas dans la formation de l'idée, les sens pourraient être malgré tout très différemment disposés quand ils sont frappés par l'objet. Il s'agit ici du quatrième mode d'Énésidème, selon lequel les perceptions varient selon les différentes dispositions

40. Huet (1974), p. 31.

41. Ibid., pp. 180-181.

42. Gassendi (1959), II, 6.

43. Sextus Empiricus (1998), I, 118-123.

44. Huet (1974), pp. 36-37. 
des sens ${ }^{45}:$ " nous sentons les choses autrement quand les organes des sens sont sains et vigoureux, et autrement quand ils sont malades. Plusieurs choses étaient à nôtre goût pendant notre enfance, qui nous paraissent dégoûtantes dans un âge avancé ${ }^{46} »$.

(3) Si l'on accepte que les variations du milieu et des sens ne créent pas de problèmes épistémologiques, les nerfs et les esprits animaux n'en sont pas moins très divers en chacun. Huet mélange ici le mode sceptique des variations avec la physiologie cartésienne. Selon le deuxième mode d'Énésidème, les différences corporelles peuvent affecter les impressions sensibles d'une même chose $^{47}$. En plus des variations perceptives résultant de la diversité anatomique et physiologique des hommes, il y a aussi des variations causées par des facteurs internes et externes. Huet associe le deuxième au quatrième mode, qui évoque les différentes conditions du sujet de la perception. Celle-ci sera très différente si le sujet est malade ou sain, ivre ou sobre, vieux ou jeune ${ }^{48}$. Huet déploie les modes pyrrhoniens à partir de sa connaissance de la science physiologique de l'époque établie par Descartes et, en conséquence, remet au goût du jour le pyrrhonisme ancien.

[L]es esprits sont quelquefois si agités par la maladie, par le sommeil, par le vin et par d'autres causes, que les fibres du cerveau en sont si violemment ébranlées que le cerveau en reçoit diverses impressions, en sorte que l'entendement pense quelquefois avoir de certains sentiments, que les organes des sens n'ont point $\mathrm{eu}^{49}$.

On ne peut que penser à la physiologie du Traité de l'Homme et à la première partie des Passions, dont l'article 14 est intitulé "Que la diversité qui est entre les esprits peut aussi diversifier leur cours ", l'article 15 examinant les causes de cette diversité :

Et cette inégalité peut procéder des diverses matières dont [les esprits animaux] sont composés, comme on voit en ceux qui ont bu beaucoup de vin que les vapeurs de ce vin, entrant promptement dans le sang, montent du cœur au cerveau, où elles se convertissent en esprits qui, étant plus forts et plus abondants que ceux qui y sont d'ordinaire, sont capables de mouvoir le corps en plusieurs étranges façons. Cette inégalité des esprits peut aussi procéder des diverses dispositions du cœur, du foie, de l'estomac, de la rate et de toutes les autres parties qui contribuent à leur production ${ }^{50}$.

On voit comment Huet dérive des conséquences épistémologiques sceptiques d'une physiologie qui n'a pas du tout cette visée chez Descartes.

45. Sextus Empiricus (1998), I, 100-117.

46. Huet (1974), p. 39.

47. «Le deuxième mode dérive des différences parmi les hommes » qui peuvent être corporelles ou relatives à l'âme; Sextus Empiricus (1998), I, 79-91.

48. Ibid., I, 100-117.

49. Huet (1974), pp. 45-46.

50. Descartes (1964), XI, p. 340. 
(4) À supposer que les fibres et les esprits soient de fidèles témoins, les cerveaux n'en resteraient pas moins variables. Encore une fois, Huet " pyrrhonise » la physiologie cartésienne à partir du deuxième mode d'Énésidème comme le montre l'extrait suivant des Passions de l'âme $(\mathrm{I}, 39)$ :

La même impression que la présence d'un objet effroyable fait sur la glande, et qui cause la peur en quelques hommes, peut exciter en d'autres le courage et la hardiesse, dont la raison est que tous les cerveaux ne sont pas disposés en même façon, et que le même mouvement de la glande qui, en quelques-uns, excite la peur, fait dans les autres que les esprits entrent dans les pores du cerveau qui les conduisent partie dans les nerfs qui servent à remuer les mains pour se défendre, et partie en ceux qui agitent et pissent le sang vers le cœur, en la façon qui est requise pour produire des esprits propres à continuer cette défense et en retenir la volonté ${ }^{51}$.

Puisque l'explication cartésienne de la perception sensible relève aussi d'un mouvement des esprits et des traces que ceux-ci causent sur le cerveau, Huet peut réhabiliter les modes pyrrhoniens à travers la science de l'homme de Descartes, détournant le contexte physiologique et moral des Passions vers un contexte épistémologique. Comme l'anatomie du cerveau n'est pas exactement la même en différentes personnes, les traces qui y sont imprimées ont alors des variations qui, bien qu'elles soient négligeables, peuvent malgré tout altérer l'image sensible de la chose.

(5) Enfin, même si le processus mécanique de la perception de (1) à (4) ne pose pas de problèmes épistémologiques, «nous ne savons [...] pas de quelle manière cette impression qui se fait dans le cerveau peut parvenir à l'âme, et que l'âme cependant se sente ébranlée et affectée en quelque façon par le cerveau, qui a été ébranlé lui-même par un mouvement corporel ${ }^{52}$. Conclusion moderne du pyrrhonisme revu et corrigé par Huet à la lumière du cartésianisme. La distinction réelle de l'âme et du corps casse la chaîne — déjà problématique - de la perception, laissant dans une obscurité complète le rapport entre l'idée et l'objet. La principale preuve sceptique que Huet exploite dans cet argumentaire renvoie au dualisme et la physiologie cartésiennes ${ }^{53}$.

\section{Le scepticisme de Huet fut perçu comme partiellement cartésien par ses premiers lecteurs (deux philosophes anti-cartésiens, amis intimes de l'évêque)}

Huet a choisi de montrer à certains de ses amis intimes le manuscrit de la version originale des Quaestiones Alnetanea, dont la première partie contient le Traité, la deuxième la Censura et la troisième la Concordia, afin de bénéficier

51. Ibid., XI, pp. 358-359.

52. Huet (1974), pp. 48-49.

53. Au milieu de ce chapitre, Huet évoque la physiologie cartésienne et manifeste son désaccord avec certains détails de cette théorie (pp. 42-43). Comme il le rappelle à ce propos, il sait de quoi il en retourne puisqu'il a fait lui-même des expériences anatomiques. Cependant, la théorie cartésienne est présentée par Huet comme en gros véritable. 
de leurs suggestions et critiques et de tester également la réaction des lecteurs en vue d'une possible publication. Elena Rapetti a publié les rapports de deux de ces lecteurs, les pères Louis le Valois, jésuite très anti-cartésien, et JeanBaptiste du Hamel, critique aussi de Descartes, bien que son statut d'oratorien le conduise à manifester moins d'hostilité que le jésuite à l'encontre du cartésianisme ${ }^{54}$. Les deux compte rendus qu'ils en font sont très négatifs en ce qui concerne le premier livre du manuscrit, le Traité. Cette réaction a vraisemblablement pesé dans la décision de Huet de ne pas le publier en compagnie des deux autres livres, le livre anti-cartésien et le livre apologétique qui ont, eux, connu un accueil beaucoup plus favorable de la part de ses amis.

Ce qui nous paraît le plus remarquable dans les réactions de Le Valois et Du Hamel vis-à-vis du Traité, c'est leur perception d'un cartésianisme sceptique chez leur ami, pourtant farouche anti-cartésien. Voici par exemple la réaction de Le Valois à l'argument du Dieu trompeur: "Le 9. argument, qui est de Descartes, me paraît choquant. Mon esprit ne s'y apprivoise point. Non plus qu'à cette proposition de la p. 27 [du manuscrit] : Fieri potest ut hominem alicubi procreauerit Deus ratione non praeditum ». Le Valois réagit ici à l'usage sceptique huétien de la doctrine cartésienne de la création des vérités éternelles ${ }^{55}$. Du Hamel semble encore plus intrigué par l'usage du doute cartésien que fait Huet. Il commence son compte rendu par un bref passage d'un manuscrit d'un docteur qu'il ne nomme malheureusement pas, qui contestait l'utilité du doute dans la philosophie, et en particulier dans l'apologétique ${ }^{56}$.

On ne manquera pas de nous objecter ce qu'un docteur a objecté autrefois contre cette suspension de Mr. Descartes. Car ce que dit Mr. Descartes, qu'il est bon pour examiner la vérité de mettre toutes choses en doute une fois dans sa vie, les sceptiques que vous défendez voulaient qu'on le fît toujours ${ }^{57}$.

Les éléments donnés dans les sections 1 à 6 témoignent de la nécessité de repenser la portée du cartésianisme à la fin du XVII siècle. Parce que l'historiographie de la philosophie moderne a relevé l'influence de la métaphysique cartésienne et laissé de côté son influence méthodologique, on ne trouve pas de difficulté à penser les philosophies de Malebranche ou de Leibniz comme des développements philosophiques originaux, mais qui appartiennent de droit $\mathrm{au}$ "cartésianisme " de cette période. Par contre, on ne pense jamais à Huet ou à Foucher comme des "cartésiens ${ }^{58}$ au même titre que les autres, à savoir non pas comme des disciples, mais comme des continuateurs/modificateurs des démarches ou doctrines du grand philosophe du XVII ${ }^{e}$ siècle.

54. Ses compte rendus serviront par la suite, cf. Louis le Valois (1680) et Jean Du Hamel (1678)

55. Cité dans Rapetti (2003), p. 74.

56. Du Hamel parle seulement d'un manuscrit qui «a tombé il y a quelques temps entre [ses] mains» $(1678$, p. 173). Je n'ai pu identifier ni le manuscrit ni son auteur.

57. Ibid.

58. Cf. Maia Neto (2003). 


\section{Bibliographie:}

Alberti, A. "Lo scetticismo apologetico di Pierre Daniel Huet ", Gionarle critico della filosofia italiana, 57, 1978, pp. 210-237.

Belgioioso, G. La variata immagine di Descartes : gli itinerati della metafisica tra Parigi e Napoli (1690-1733), Lecce, Milella, 1999.

Borghero, C. «Discussioni sullo scetticismo di Descartes (1650-1712) », Giornale Critico della Filosofia Italiana, 6, 18, 1998, pp. 1-25.

Descartes, R. Lettres de Mr. Descartes, Paris, Charles Angot, 1667.

-. Euvres, Paris, Vrin, 1964-1974, 11 vol.

Diogène Laërce, Vies et doctrines des philosophes illustres, Paris, Librairie générale française, 1999.

Du Hamel, J. Philosophia vetus et nova ad usum scholae accomodata, Paris, 1678.

Dupront, A. Pierre-Daniel Huet et l'exégèse comparatiste, Paris, 1930.

Gassendi, P. Dissertations en forme de paradoxes contre les aristotéliciens, Paris, Vrin, 1959.

Huet, P.-D. Censura philosophiae cartesianae, Paris, D. Horthemels, 1689.

—. Alnetanea quaestiones de concordia rationis et fidei, Caen, De Cavelier, 1690.

—. Traité philosophique de la foiblesse de l'esprit humain, Hildesheim, Olms, 1974 (reproduction anastatique de l'édition d'Amsterdam, 1723).

—. Mémoires, Toulouse, Société de Littératures Classiques, 1993.

—. «La censure de la réponse faite par M. Regis au livre intitulé Censura philosophiae cartesianae", Bibliothèque Nationale de France, manuscrit Fonds Français 14703, n. 3.

- Correspondance manuscrite de Nicaise, Bibliothèque Nationale de France, manuscrit Fonds Français 9359, foll. 108.

Lennon, T. «Huet, Malebranche and the Birth of Skepticism », dans G. Paganini, dir., The Return of Scepticism from Hobbes and Descartes to Bayle, Kluwer, Dordrecht, 2003, pp. 149-165.

—_. "The Skepticism of Huet's Traité philosophique de la foiblesse de l'esprit humain », dans M. A. Bernier et S. Charles, dir., Scepticisme et Modernité, SaintÉtienne, Publications de l'Université de Saint-Étienne, 2005, pp. 65-75.

Le Valois, L. Sentiments de M. Descartes touchant l'essence et les propriétés $d u$ corps, opposés à la doctrine de l'Église, et conformes aux erreurs de Calvin, sur le sujet de l'Eucharistie, Paris, 1680.

Lux, D. Patronage and Royal Science in Seventeenth-Century France: the Académie de Physique in Caen, Ithaca, Cornell University Press, 1989.

Maia Neto, J. "'As principais forças dos pirrônicos' (Lafuma 131) e sua apropriação por Huet ", Kriterion, 47, 114, 2006, p. 247-257.

- - "Foucher's Academic Cartesianism ", dans T. Lennon, dir., Cartesian Views. Papers presented to Richard A. Watson, Leiden, Brill, 2003, p. 71-95.

Malbreil, G. «Les droits de la raison et de la foi, la dissociation de la raison, la métamorphose de la foi, selon Pierre Daniel Huet », XVII Siècle, 37,2, 1985, p. 119133.

—. "Le Traité philosophique de la foiblesse de l'esprit humain, de feu Monsieur Huet, ancien évêque d'Avranches ", dans S. Guellouz, dir., Pierre-Daniel Huet (1630 - 1721), Actes du Colloque de Caen (1993), Paris, Biblio 17, 1994, p. $169-182$.

Massimi, J.-R. "Vérité et histoire chez Pierre-Daniel Huet ", XVII Siècle, 37,2, 1985, p. 167-168. 
Pélissier, L. Lettres inédites de Claude Nicaise à Huet et à G. Bonjour, Dijon, impr. De l'Union, 1889.

Quantin, J.-L. «La Raison, la Certitude, la Foi : quelques remarques sur les préliminaires de l'acte de foi selon Huet ", dans S. Guellouz, dir., Pierre-Daniel Huet (1630 - 1721), Actes du Colloque de Caen (1993), Paris, Biblio 17, 1994, p. 8397.

Rapetti, E. Percorsi anticartesiani nelle lettere a Pierre-Daniel Huet, Florence, Leo S. Olschki, 2003.

Sextus Empiricus, Hypotyposes pyrrhoniennes (traduction Pierre Pellegrin), Paris, Seuil, 1998.

Tolmer, L. Pierre-Daniel Huet : humaniste-physicien, Bayeux, Colas, 1949. 